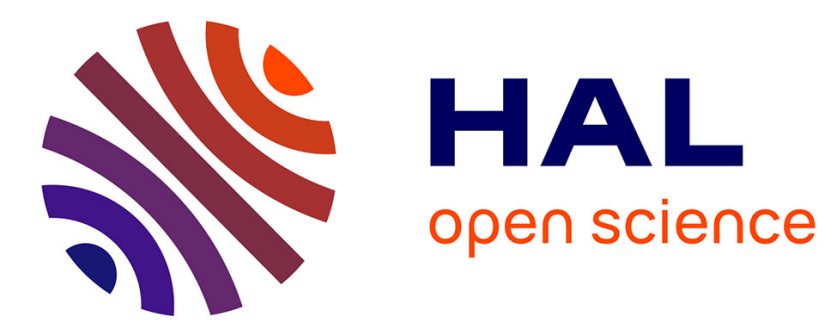

\title{
Boisement de terres libérées par l'agriculture Jean Gadant
}

\section{- To cite this version:}

Jean Gadant. Boisement de terres libérées par l'agriculture. Revue forestière française, 1989, 41 (6), pp.463-467. 10.4267/2042/25997 . hal-03425274

\section{HAL Id: hal-03425274 https://hal.science/hal-03425274}

Submitted on 10 Nov 2021

HAL is a multi-disciplinary open access archive for the deposit and dissemination of scientific research documents, whether they are published or not. The documents may come from teaching and research institutions in France or abroad, or from public or private research centers.
L'archive ouverte pluridisciplinaire HAL, est destinée au dépôt et à la diffusion de documents scientifiques de niveau recherche, publiés ou non, émanant des établissements d'enseignement et de recherche français ou étrangers, des laboratoires publics ou privés. 


\title{
BOISEMENT DE TERRES LIBÉRÉES PAR L'AGRICULTURE
}

\author{
J. GADANT
}

Depuis que la Communauté économique européenne limite les productions agricoles, on s'interroge sur le devenir des terres que cette "déprise agricole" voue à l'abandon. Une récente publication, au titre excessif, retient actuellement l'attention des médias : "La France en friche".

Diverses alternatives sont proposées: l'agriculture extensive consommatrice d'espace, la rémunération de l'engagement de non produire et la jachère verte, le retour à l'état naturel des terres les moins fertiles, leur aménagement à des fins cynégétique, écologique ou touristique, enfin la création de peuplements forestiers.

Nous pensons que la solution du boisement, parfois curieusement contestée, constitue une opportunité dont la politique forestière devrait se saisir, moyennant un strict cadrage des réalisations et la mise en œuvre de mesures d'accompagnement.

En effet, la forêt représente une séduisante possibilité d'utilisation des terres que l'agriculture abandonne; elle constitue aussi un bon créneau pour contribuer au développement de certaines zones rurales déshéritées, en voie de désertification, mais qui sont aptes à une production ligneuse de haut rendement: basses montagnes, plateaux granitiques du Limousin et de l'Auvergne, bordure sud du Massif Central, massif du Morvan, etc...

II était donc logique que l'idée de boisement soit associée à cette situation de déprise agricole. D'ailleurs, la reforestation de terres que l'agriculture délaisse n'est pas une préoccupation nouvelle; depuis la dernière guerre, elle accompagne l'exode rural et le recul des activités agricoles.

Néanmoins, l'engagement d'une telle politique de boisement fait l'objet d'interrogations, d'hésitations, parfois même de réticences et d'une grande prudence que le ministre de l'Agriculture manifestait dans sa déclaration de politique forestière du 18 tévrier 1987 : "La forêt pourra sans doute apporter une contribution positive au problème de l'abandon prévisible de certaines terres agricoles. Mais j'insisterai sur la nécessaire prudence et la progressivité indispensable liées à une telle entreprise".

Ces réticences, parfois I'hostilité de certains, paraissent fondées sur deux types d'arguments, hors la crainte d'une baisse des cours des bois qui deviendraient plus abondants:

- des forestiers sont hésitants, alors que les prix des bois stagnent et que les boisements qui ont èté créés, depuis quarante ans, peuvent poser, dans quelques cas, des problèmes de débouchés ;

- d'autres pensent qu'il serait plus judicieux d'intensifier la gestion du patrimoine forestier actuel, plutôt que de procéder à son extension sur de nouveaux espaces. 


\section{J. GADANT}

À terme, ces arguments sont cependant contestables. En effet, l'Europe manque considérablement de bois et les importations qui sont faites pour combler le déficit ont de très lointaines provenances; de plus, selon les experts, l'écart se creuse au niveau mondial entre l'accroissement des besoins et la capacité du potentiel forestier actuel à les satisfaire. Par ailleurs, dans un espace économique européen, industrialisé et urbanisé, avec des zones de forte densité démographique, si demain le bois devenait matériau inutile, la forêt conserverait de multiples fonctions vis-à-vis desquelles notre société se montrera de plus en plus exigeante: la forêt modératrice des climats, protectrice des sols et régulatrice du débit des eaux, notamment en montagne ; la forêt oxygénante, dépoussiérante et bactéricide; la forêt espace de promenade et de loisirs; la forêt paysagère, etc... Faudrait-il encore que tous ces précieux services, dont l'intérêt va grandissant, ne demeurent pas gratuits pour les sylviculteurs.

Peut-être a-t-on mal posé le problème ? II est certain que l'idée de boisement qui aurait pour objet de régler un problème agricole et de s'opposer à la friche est simpliste. L'idée d'envisager le boisement des terres gelées par la politique agricole commune est également stupide car ce gel est provisoire et les parcelles concernées sont dispersées. Par ailleurs, certains ont pu redouter qu'une forêt étouffante déferle, en désordre, sur des millions d'hectares libérés. Sans doute, convient-il aussi de cadrer une ambition forestière nouvelle par des conditions limitatives au plan des réalisations: terres suffisamment productives (la forêt n'a pas vocation à occuper les espaces que les autres activités abandonnent), unité de gestion de taille suffisante (pour faire de la vraie forêt (étendue et équipée), facile à gérer et à exploiter).

Mais d'un strict point de vue de politique forestière, il nous paraît que le moment est venu de poursuivre résolument, voire d'intensifier la politique de reboisement engagée en 1946 avec la création du Fonds forestier national.

Il y a d'abord une raison nationale : l'achèvement du programme en cours de boisement engagé en 1946. En effet, une politique de reboisement doit se réaliser de façon continue, à des cadences annuelles aussi constantes que possible, durant une révolution de peuplement (du semis à l'exploitation), afin d'assurer la pérennité de la ressource, de ne pas créer un déséquilibre des classes d'âge et des difficultés d'approvisionnement pour les industries qui auront été mises en place. L'objectif initial portait sur 3 millions d'hectares, à réaliser sur une période de 60 ans, à la cadence de 50000 hectares par an; il reste donc à poursuivre une politique de reboisement sur une période de 20 ans à la cadence de 50000 hectares par an. Ainsi, il s'agit d'achever le programme de reboisement entrepris après la dernière guerre, pour la période 1950-2010, plutôt que d'engager une nouvelle campagne. Les terres que l'agriculture abandonne constituent aujourd'hui une opportunité favorable à l'achèvement de cette politique, tandis que cette exigence de politique forestière apporte une contribution positive au développement de territoires ruraux gravement affectés par les abandons de terres agricoles.

Ensuite il y a une raison européenne: le déficit européen en bois. Dans une Europe où les surproductions agricoles font problème, l'énorme déficit en bois interroge. Trois chiffres éloquents se passent de commentaires pour justifier l'intérêt d'une politique européenne de reboisement dans laquelle la France pourrait jouer un rôle moteur important :

- La Communauté économique européenne est le deuxième importateur mondial de bois : elle importe 90 millions de mètres cubes sur une consommation de 190. La valeur de ce déficit atteint 15 milliards d'écus.

- La consommation de bois par Européen est encore modeste : de l'ordre de $0,6 \mathrm{~m}^{3}$, alors qu'elle dépasse $2 \mathrm{~m}^{3}$ aux États-Unis et atteint $1,25 \mathrm{~m}^{3}$ au Japon.

- Le patrimoine forestier européen ne représente que 1,2\% des forêts mondiales et la surface forestière par Européen est modeste $(0,16$ ha), alors qu'elle dépasse 2 ha en Amérique et atteint 3 ha en URSS. 
Ces chiffres engagent donc à promouvoir, dans cette Europe très déficitaire, un développement des espaces forestiers et des industries du bois. La France y tient une place particulièrement favorable: elle est la plus riche en espace; elle dispose de terroirs aptes à une production ligneuse de qualité et de haut rendement : une politique de reboisement, vieille de quarante ans, lui a donné de l'expérience et du savoir-faire dont l'Europe pourrait tirer profit.

Deux documents de grande importance élaborés par la Commission économique européenne et soumis actuellement à l'examen du Conseil des Ministres paraissent donc étroitement liés: l'avenir du monde rural et l'action forestière de la Communauté économique européenne. Le document de la CEE du 23 septembre 1988 intitulé "Stratégie et actions de la Communauté dans le secteur forestier "va dans ce sens en proposant le boisement des terres agricoles et une mise en valeur plus intensive des forêts. Plus récemment, la Présidence espagnole avançait quelques propositions conformes: "amélioration de l'autosuffisance en bois de l'Europe, récupération et reboisement de terres agricoles, etc... "

II y a, enfin, une raison internationale: la mondialisation du marché du bois. La Communauté européenne importe des sciages résineux de Scandinavie, d'Europe centrale, du Canada; des bois tropicaux d'Afrique et du Sud-Est asiatique; de la pâte à papier d'Amérique du Nord et de Scandinavie. Or les études des experts du Comité du Bois de Genève sur l'évolution du marché du bois, à l'horizon de l'an 2000, concluent que la demande en bois ira en s'accroissant, tandis que la Communauté internationale manifeste de l'inquiétude sur la régression importante des grands espaces forestiers, notamment tropicaux.

Pour réaliser une telle politique d'accroissement de la production de bois, il existe plusieurs scénarios sylvicoles: arboriculture forestière, taillis intensifs traités à courte révolution, boisements traditionnels semi-extensifs. Le choix devrait porter sur des boisements les moins artificialisés possibles ; il devrait aussi privilégier l'objectif " boís d'œuvre " dont la production génère des volumes importants de petits bois d'éclaircie et de produits connexes de scieries utilisés dans les industries de la trituration.

Les boisements du passé ne sont pas exempts de critiques: stations insuffisamment productives, dispersion, timbres-poste, enrésinements de feuillus parfois contestables. L'objectif est de créer de la forêt facile à équiper (desserte), à gérer et à exploiter (étendue suffisante). Pour y parvenir, de strictes conditions doivent s'imposer à ces réalisations de boisement :

- Être conçu, étudié et programmé dans le cadre d'un plan d'aménagement et de développement local. Ce dernier aura permis de sensibiliser les propriétaires, de localiser les bonnes zones à boiser, de dresser un avant-projet. II pourrait même être envisagé de privilégier ces zones dans l'attribution des aides financières.

- Se placer à l'aval de vigoureuses opérations de restructuration foncière car les terres disponibles seront dispersées, morcelées, imbriquées avec des activités agricoles maintenues. Mais la France vient de se doter des outils d'aménagement foncier nécessaires: zonage agriculture-forêt, remembrement forestier, réorganisation foncière agricole et forestière, aménagement foncier agricole et forestier.

- Concurremment avec les initiatives privées qui ont été déterminantes dans le passé, responsabiliser davantage les collectivités locales qui pourraient être appelées à jouer un rôle important dans cette politique. En France, la forêt publique ne représente que le quart du patrimoine forestier national, alors qu'elle représente plus de $40 \%$ en Belgique, GrandeBretagne, Allemagne, Grèce, Irlande, Italie, Luxembourg et Hollande.

- Privilégier davantage les exploitants agricoles qui pourraient devenir d'actifs reboiseurs, gestionnaires de forêts et exploitants forestiers s'ils sont encadrés et aidés. Ces travaux forestiers apporteraient des revenus d'appoint et pourraient contribuer au maintien en activité d'exploitations agricoles marginales appelées à disparaître. Dans le passé, forêt et agriculture ont été concurrentes; elles deviendront ainsi plus complémentaires. 


\section{J. GADANT}

Mais ce reboisement dont la nécessité vient d'être démontrée devra être accompagné d'autres politiques, notamment :

- le développement des industries de transformation du bois, afin que les volumes actuellement disponibles soient assurés de débouchés. Mais il faut aussi se convaincre que cet effort industriel nécessaire sera d'autant mieux assuré que les investisseurs disposeront d'une garantie d'approvisionnement à long terme. II faut sortir du cercle vicieux: je ne plante pas car les débouchès ne sont pas assurès; je n'investis pas car les approvisionnements industriels ne sont pas garantis.

- la poursuite de l'objectif de conversion et d'amélioration des forêts insuffisamment productives. En effet, le choix sélectif des terres à boiser, la constitution d'unitès de gestion de taille suffisante, les délais d'exécution, limiteront les surfaces des terres agricoles éligibles au boisement.

En conclusion, l'énorme déficit en bois de l'Europe, la valorisation des potentialitès forestières de zones en voie d'abandon, l'aménagement du territoire rural et du cadre de vie militent fortement en faveur d'une redynamisation de la politique nationale de reboisement. En tirant la synthèse des travaux du Conseil supèrieur de la Forêt, en sa séance du 29 mars 1989, le ministre Henri Nallet prônait cette relance maîtrisèe de la politique forestière française. Laissons lui donc le dernier mot: "Parce que nous sommes le plus grand pays forestier de la Communauté, nous devons jouer un rôle militant dans l'élaboration d'un projet forestier européen. La France est bien placée pour jouer ce rôle de leader dans la formulation de cette politique car elle dispose d'une avance conceptuelle et d'un capital d'expérience d'une exceptionnelle richesse".

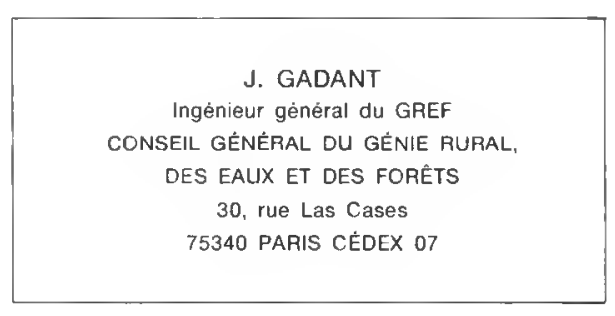




\section{BOISEMENT DE TERRES LIBÉRÉES PAR L'AGRICULTURe (Résumé)}

Dans une Europe très déficitaire en bois, les terres que les excédents agricoles vnuent à l'abandon offrent une opportunité de relance de la politique de reboisement engagée en 1946 avec la création du Fonds forestier national. Par ailleurs, l'aménagement du territoire rural et du cadre de vie impose un effort accru de reforestation afin de faire échec à la friche.

Les nouvelles plantations sont à concevoir et a programmer dans le cadre de plans d'amenagement rural et de vigoureuses opérations de restructuration foncière. En effet, les terres devant se libérer sont dispersées et imbriquées avec les activités agricoles maintenues. II importe de créer des forêts d'étendue suffisante, bien équipées en dessertes, faciles à gèrer ê à exploiter.

Par ailleurs, il est essentiel qu'un effort de développement des industries du bois, d'intensification de la gestion et de conversion des mauvais peuplements feuillus accompagne cette politique d'extension forestière.

\section{AFFORESTATION OF LAND RELEASEO BY AGRICULTURE (Summary)}

In a situation of severe timber shortage in Europe, the land abandoned as a result of agricultural surpluses provides an opportunity for reviving the afforestation policy initiated in 1946 with the creation of the French National Forestry Fund. However, rural development means that afforestation efforts must be increased in order to prevent land becoming fallow.

In theory, new plantations should be incorporated into rurai development plans and radical land reorganisation operations. In fact, the areas to be released are scattered and split between areas still used for agriculture. It is essential that the forests created are of a reasonable size with good communication networks and are easy to manage and exploit.

However, this afforestation policy must be accompanied by measures to develop forest industries, by more intensive management and conversion of poor broadleaved stands.

\section{AUFFORSTUNG DER VON DER LANDWIRTSCHAFT NICHT MEHR GENUTZTEN FLÄCHEN (ZusammenfosSUng)}

Angesichts des europäischen Holzdefizits, bieten die aufgrund der Agrarüberschüsse brachliegenden Böden, eine Gelegenheit zur Wiederankurbelung der 1946 mit der Schaffung des Nationalen Forstfonds begonnenen Aufforstungspolitik. Andererseits fordert die ländliche Raumordnung unt Neugestaltung des Lebensraumes eine erhöhte Anstrengung auf dem Gebiet der Aufforstung um brachliegende Flächen zu vermeiden.

Die Neubepflanzungen müssen im Rahmen der ländlichen Raumordnungspläne und bedeutender Maßnahmen der Flurbereinigung konzipiert und geplant werden, denn die freiwerdenden Böden liegen verstreut und eingeschlossen in den weiterhin landwirtschaftlich genutzten Flächen. Es ist aber notwendig Wälder von einem gewissen Mindestausmaß zu schaffen, mit guten Verbindungswegen, die zudem leicht zu bewirtschaften sind.

Es ist außerdem von großer Bedeutung, daß Anstrengungen zur Entwicklung der Holzindustrie, der Intensivierung der Bewirtschaftung und der Erneuerung der schlechten Laubbestände diese Politik der Forstausdehnung begleiten.

\section{REPOBLACION DE TIERRAS LIBERADAS POR LA AGRICULTURA (Resumen)}

En una Euroda muy deficiteria en bosques, las tierras que los excedentes agrícolas destinan al abandono ofrecen una oportunidad de reactivación de la politica de repoblación, emprendida en 1946 con la creación del Fondo Forestal Nacional. De otra parte, la estructuración del territorio rural y del cuadro de vida impone un esfuerzo creciente de la reforestación, a fin de evitar los yermos.

Las nuevas plantaciones deben ser concebidas y programadas en el cuadro de los planes de acondicionamiento rural y de vigorosas operaciones de reestructuración, fundamentales. En efecto, las tierras que deben liberarse están dispersas e imbricadas con las actividades agricolas mantenidas. Es preciso crear bosques de suficiente extensión, aunque bien equipadas en servicios, de fácil gestión y explotación.

Desde luego, es esencial que esta politica de extensión forestal sea acompañada de un esfuerzo de desarrollo de las industrias madereras, de una intensificación de la gestión y de la conversión de las malas replobaciones de frondosos. 